\title{
Danza Pobre: Una reflexión sobre el surgimiento de la danza moderna en Los Estados Unidos
}

\author{
Sarahí Lay Trigo \\ University of California - Santa Cruz, CA/EUA \\ E-mail: sarahilaytrigo@gmail.com
}

\section{Resumen}

Haciendo alusión a la obra clásica Hacia un Teatro Pobre de Jerzy Grotowski (2004) y su Laboratorio Teatral, este artículo considera el surgimiento de la danza moderna en los Estados Unidos como una forma de hacer danza pobre. Esto es así porque el movimiento de transformación impulsado por los creadores de este estilo dancístico ofrece increíbles similitudes con los principios que formularía después Grotowski para los actores. Así pues, el teatro pobre como la danza moderna, a pesar de haber surgido en diversos espacios y tiempos, son dos movimientos análogos que comparten, entre otras cosas, la forma de hacer y concebir el arte. Sus mayores similitudes se encuentran en los siguientes principios: 1) El arte como vía negativa; 2) El arte como pobreza; y 3) El arte como encuentro espiritual. Para llevar a cabo esta analogía se utilizará una perspectiva histórico-biográfica en dos niveles. Uno, el histórico, que sirve para ofrecer un panorama general del contexto social que favoreció esta nueva creación dancística. Dos, el biográfico, que aborda brevemente algunos momentos de la vida de tres de los principales creadores de la primera generación de la danza moderna, a saber, Isadora Duncan, Ruth St. Denis y Ted Shawn.
Com uma sugestão do clássico de Jerzy Grotowski (2004) Towards a Poor Theatre e seu Laboratório Teatral, este artigo considera a ascensão da dança moderna nos Estados Unidos como uma maneira de fazer dança pobre. Isso ocorre porque o movimento de transformação conduzido pelos criadores desse estilo de dança oferece incríveis semelhanças com os princípios que Grotowski mais tarde formularia para os atores. Assim, o teatro pobre como a dança moderna, apesar de ter surgido em diferentes espaços e tempos, são dois movimentos semelhantes que compartilham, entre outras coisas, o modo de fazer e conceber a arte. Suas maiores semelhanças são encontradas nos seguintes princípios: 1) A arte como rota negativa; 2) Arte como pobreza; e 3) arte como encontro espiritual. Para realizar essa analogia, será usada uma perspectiva histórico-biográfica de dois níveis. Um, o histórico, que serve para oferecer um panorama geral do contexto social que favoreceu essa nova criação da dança. Segundo, o biográfico, que aborda brevemente alguns momentos da vida de três dos principais criadores da primeira geração da dança moderna, a saber, Isadora Duncan, Ruth St. Denis e Ted Shawn. 


\section{Obertura}

La historia de algo es la esencia de ese algo. Es en la historia de la danza donde debemos buscar la esencia de su significado social y político. (SOTO, 2008, p. 34).

Hacer una analogía entre dos movimientos artísticos como el surgimiento de la Danza Moderna en los Estados Unidos ${ }^{1}$ a principios de siglo $X X$ y el Teatro Pobre (Laboratorio Teatral) ${ }^{2}$ a mitad de siglo (en la década de los sesenta) en Polonia, requiere de una mirada reflexiva e histórica que permita dar luz sobre sus semejanzas. Para indagar en ello, este trabajo se apoya en el método histórico-biográfico en dos dimensiones o momentos.

El primer momento tiene como propósito visibilizar algunos aspectos del contexto social que sirvieron para impulsar e inspirar el surgimiento de la danza moderna en los Estados Unidos. Aquí, la reflexión tiene como eje principal del teatro pobre el principio denominado método de eliminación.

En un segundo momento, para ver cómo es que los cambios históricos comienzan por gestarse en los individuos, se retoman, de manera concisa, algunos fragmentos biográficos de la vida de Isadora Duncan, Ruth St. Denis y Ted Shawn. La reflexión se detiene en estos artistas de la primera generación y no en otros por dos razones. Primero, porque con sus historias es posible encontrar con mayor facilidad algunos de los principales fundamentos del teatro pobre, a saber, el arte como pobreza y el arte como en-

1 No se entienda por esto que en este artículo se afirma que la Danza Moderna surgió exclusivamente en Estados Unidos. Para profundizar en ello ver Anderson (1997).

2 “En 1959 Jerzy Grotowski creó el Laboratorio Teatral en Opole, ciudad de 60000 habitantes en el suroeste de PoIonia. El conocido crítico literario y teatral Ludwik Flaszen, su colaborador íntimo, lo ayudó en esa tarea. En enero de 1965, el laboratorio Teatral se mudó a la ciudad universitaria de Wroclaw, que con su medio millón de habitantes es la capital cultural de los territorios orientales de Polonia. En Wroclaw se convirtió en el Instituto de Investigación del Actor. La actividad del Laboratorio ha recibido desde entonces el subsidio oficial a través de las municipalidades de Opole y de Wroclaw" (GROTOWSKI, 2004, p. 3). cuentro espiritual. Y segundo, porque su aportación a la danza moderna fue definitiva, tanto para su desarrollo como para su difusión en los Estados Unidos.

Aquí es importante señalar que las creaciones dancísticas de otros protagonistas en los Estados Unidos como Loïe Fuller, Maud Allan (de la primera generación); Martha Graham, Doris Humprey, Charles Weidman, José Limón, Lester Horton, Anna Sokolow (pertenecientes a la segunda generación); Merce Cunningham, Paul Taylor, Alwin Nikolais y Alvin Ailey (de la tercera generación), -por señalar algunostambién fueron de significativa importancia para el surgimiento y establecimiento de la danza moderna. Sin embargo, esto queda pendiente para otro momento, ya que el propósito principal de este trabajo no radica en rescatar con detalle la evolución histórica de esta manifestación artística, sino detenerse -aunque de manera sucinta- en aquellas similitudes que la danza moderna de principios de siglo XX comparte con el teatro pobre. Para realizar el análisis y reconocer cómo es que ciertos principios del teatro pobre están presentes en el surgimiento de la danza moderna norteamericana, el trabajo se divide de la siguiente manera, I) El arte como vía negativa: Contexto histórico; II) El arte como pobreza: Isadora Duncan; y III) El arte como encuentro espiritual: Ruth St. Denis y Ted Shawn. A continuación se desarrollan.

\section{El arte como vía negativa: contexto histórico}

La nuestra es una vía negativa, no una colección de técnicas, sino la destrucción de obstáculos. [...] Ésta es la diferencia esencial [...] no es una técnica positiva. No queremos recetas, estereotipos, prerrogativa de los profesionales. (GROTOWSKI, 2004, p. 11-178).

Mucho antes que la vía negativa fuera un principio esencial para el teatro pobre, lo fue para el surgimiento de la danza moderna. Esta acción de «eliminar» le permitió a los pioneros del movimiento moderno en la danza realizar un proceso de «exclusión», «separación», «diferenciación» y «destrucción» de lo establecido previamente en el mundo de la danza. En este caso, realizar un proceso de 
alta diferenciación entre lo que formaría parte de su propuesta como bailarines modernos y la escuela clásica. Esto es así porque para crear algo nuevo es necesario ir en contra de lo que se instituyó primero. De modo que, cuando surgen nuevas instituciones, nuevas formas de entender el mundo "se observa que una institución toma el lugar de otra a la que trata de destruir y que ciertas maneras de ser, de pensar, siempre son expresiones que de algún modo eliminaron otras formas de pensar o de existir" (ENRÍQUEZ, 2002, p. 45). Si se sigue esto, habría que decir que para crear hace falta «destruir». Entiéndase esta acción de destrucción no como el acto formal de aniquilar algo ahí donde esté -material y físicamente- sino más bien de realizar un acto de erradicación ideológica, es decir, eliminar de las fuentes de creatividad del creador toda norma, regla o estilo pre-establecido para poder crear -en la medida de lo posible- algo distinto. Esto, en esencia, fue lo que les permitió encontrar nuevas formas de danzar. Hablemos de alquimia, de transformar lo conocido, de romper el modelo y llevar toda capacidad creadora a la conquista de nuevos horizontes.

En este caso, para que surgiera la danza moderna en Estados Unidos fue necesario crear un estilo dancístico que de alguna manera «eliminara» lo instituido por la danza clásica. Hay que tener presente que, para ese momento la danza clásica estaba diseminada e institucionalizada en gran parte del mundo (Europa, Rusia, Cuba, México, entre otros) y no sólo representaba una forma de arte, sino una forma de pertenecer al mundo de lo legitimo, de lo aceptado, una forma de hacer danza que certificaba pertenencia. Por eso fue tan importante para los pioneros de la danza moderna crear nuevas formas para la creación dancística. Sin embargo, la búsqueda de nuevos rumbos no fue fácil, a continuación se desarollan brevemente dos obstáculos que los primeros bailarines de la danza moderna tuvieron que vencer para poder crear esta nueva manera de danzar.

\section{I.1 Primer obstáculo: ir en contra de la danza hegemónica}

La danza moderna surgió en 1900 como una protesta contra la vieja sociedad y el estancamiento artístico del ballet clásico que se percibió como un entretenimiento superficial, austero, mecánico y estrechamente sostenido por formas fijas y convencionales. (MARIJAN, 2018, s.p.) ${ }^{3}$.

La danza moderna es consecuencia de los cuestionamientos, preocupaciones, inquietudes y acciones que se pusieron en movimiento durante las primeras décadas del siglo XX. Así, en América del Norte, con la llegada del vigésimo siglo se comenzaron a gestar movimientos de rebelión y/o reformación que buscaban romper normas, reglas, valores establecidos y sistemas simbólicos. Entre ellos no sólo los protagonizados por la danza sino también por las bellas artes y la vida sociopolítica de los individuos. Eso favoreció -en el seno de lo artístico- el nacimiento del arte moderno norteamericano; y en lo social el surgimiento de movimientos como el feminismo ${ }^{4}$, el pacifismo ${ }^{5}$, y el antiracismo ${ }^{6}$, por señalar algunos de los principales. En el contexto general del arte se puede señalar que,

antes de la Primera Guerra Mundial, la conciencia artística estadounidense todavía estaba detrás de los emocionantes desarrollos artísticos en Europa. Hasta 1908, un grupo de pintores que se llamaban a sí mismos the Eight -también conocidos como la Escuela de Ashcan-, escandalizaban a los neoyorquinos con sus lienzos realistas e indignos. Más tarde, durante las décadas de 1920 y 1930, pintores como George Bellows y Edward Hopper se mostraban beligerantes en su forma de representar [...] lo cotidiano de la vida. [...] Pero todos estos intentos fueron

3 Traducción propia del texto original en inglés. (En adelante Trad. Propia).

4 Para profundizar más en esto ver Keetley y Pettigrew (2005).

5 Ver Burns, 1987.

6 Sobre esto se puede consultar Reid (1949). 
eclipsados por el gran evento en la historia del arte estadounidense que tuvo repercusiones de gran alcance: el Armory Show [...] Fue como una gran batalla ganada, ya que ganó un nuevo continente para las artes. [...] La influencia artística del Armory Show en los propios artistas no fue inmediata. Pero su impacto fue de una intensidad duradera. El grito de guerra fue contra el institucionalismo. [...] La danza también hacía sus propias declaraciones confusas y contradictorias. The Rite of Spring y Parade de Diaghilev intentaron dar sentido a las ansiedades del futuro. Anna Pavlova hizo piruetas en todo el mundo despertando una belleza soñadora que no debía morir. Michel Fokine, en 1914 en una breve estadía de regreso a la compañía de Diaghilev -antes de decidirse por Nueva York- formuló una vez más las ideas fundamentales de su reforma en una carta que envió al London Times. Allí reiteró que uno debe «crear en cada caso una nueva forma de movimiento que corresponda al tema» y abandonar «la combinación de pasos preparados y establecidos» [...]. Fue en el mismo año, 1914, que Ruth St. Denis se encontró con un antiguo former divinity student ${ }^{7}$, Ted Shawn, quien compartió su creencia sobre el mensaje de la religión en la danza. Juntos fundaron una escuela y compañía que se conoció como Denishawn. (SORELL, 1981, p. 370-374). ${ }^{8}$

Como se puede advertir en la cita anterior, el contexto histórico que acompañó el periodo moderno fue sin duda un espacio que dio lugar a muchos cuestionamientos respecto a las instituciones que dictaban las normas de la vida social. En la danza, por ejemplo, se comenzó a criticar el ballet y llegó a ser considerado "por los críticos modernistas como un arte sometido rígidamente por reglas, un arte mecánico y sin alma. Esta caracterización del ballet apareció una y otra vez en las palabras y publicaciones de artistas, críticos y teóricos de la danza-

7 La traducción literal al español de former divinity student sería, antiguo estudiante de la divinidad, misma que es poco feliz, por eso se dejó la expresión en el idioma original del texto.

8 Trad. Propia.

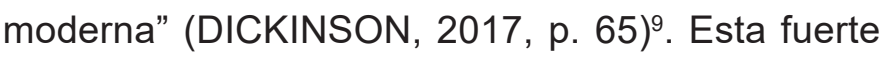
crítica favoreció el surgimiento de la danza moderna y lo caracterizó como un movimiento que buscaba,

una gran liberalización de las formas rígidas de la receta clásica, animada por el deseo de dar a la danza la calidez y vitalidad de la emoción humana. [...] Al llevar a cabo este propósito dejó de lado todo lo que había sucedido antes y comenzó de nuevo desde el principio. Este comienzo desde el principio fue el descubrimiento de la sustancia real de la danza, con este descubrimiento, la danza se convirtió por primera vez en un arte independiente, -un arte absoluto, como les gusta decir en Alemania- completamente auto-contenido [autónomo], relacionado directamente con la vida, sujeto a una infinita variedad. (MARTIN, 1965, p. 3-7)..$^{10}$

Por lo general se dice que la danza moderna comenzó, -muy a la manera del método de eliminación del teatro pobre- cuando Isadora Duncan "y otras mujeres muy activas e incluso extravagantes «mandan a volar» las zapatillas, las vestimentas, las historias fantásticas y cortesanas del ballet clásico para hacer otra forma de danza más natural" (DALLAL, 1979, p. 27). Sin embargo, eso no era lo único que se buscaba eliminar con la creación de esta nueva forma de expresión artística. No bastaba con la sola eliminación de la forma. Dicho de otro modo, no era suficiente con reemplazar las formas técnicas del movimiento clásico por otras más naturales; eliminar las zapatillas para bailar con pies descalzos; excluir los vestuarios fastuosos y extravagantes del ballet y reemplazarlos con vestuarios radicalmente distintos; quitar las historias de príncipes y princesas en mundos mágicos y bosques encantados y cambiarlas por otras más cercanas a la realidad humana. Había que ir más allá. Había que eliminar la forma «clásica» y explorar la coreografía como creación personal artística y no como una imposición inamovible.

\author{
9 Trad. Propia. \\ 10 Trad. Propia.
}




\section{I.2 Segundo obstáculo: de la danza como forma de sometimiento a la danza como camino para descubrir la vida}

Si estos bailarines rechazan las reglas del sistema clásico, lo hacen a sabiendas, con el fin de dar libre curso a su inspiración y expresar con su danza un talante propio, sin otra preocupación que la de vivir su danza. (BARIL, 1987, p. 14).

Cuando emergen los primeros exponentes de la danza moderna no se le denominó «moderna» a este estilo dancístico, sino danza libre, puesto que "durante algún tiempo, el calificativo de «libre» se da a todo tipo de formas de danza distintas a la danza teatral11" (BARIL, 1987, p. 13). Por eso, en un primer momento Isadora Duncan y Ruth St. Denis son entendidas como bailarinas libres. Esta manera de concebir la danza como libre le sirvió a los pioneros del movimiento moderno para liberarse de las exigencias y el sometimiento de la danza clásica, en particular la que se refiere al bailarín como instrumento, como "objeto» al servicio de las ideas coreográficas impuestas por una sola entidad (sea coreógrafo, maestro y/o director, por señalar los principales). Para los modernos el bailarín no podía ser, bajo ninguna circunstancia, objeto de utilización. Para los modernos el bailarín era una persona creadora. Es así como la danza moderna busca la construcción de una danza en la que el bailarín pudiera pensar-se, buscar-se, encontrar-se a sí mismo y tener un sentido distinto a lo ya instituido por la escuela clásica.

Esta idea impulsó a los pioneros de la danza moderna a colocar la figura del bailarín como artista y no como instrumento de sumisión. De manera que la danza debía estar al servicio del artista y no viceversa. Con esto se le devolvió a la danza su función de descarga expresiva emocional, psíquica y corporal. Se "reanuda así (después de cuatro siglos de «ballet clásico» y veinte siglos de desprecio del cuerpo a causa de un cristianismo pervertido por el dualismo

11 Cuando el autor habla de danza teatral se refiere a la danza que tiene como base la técnica clásica. platónico) lo que significó la danza [...] en todos los tiempos: la expresión" (GARADUDY, 2003, p. 15). Con la expresión, esta forma de hacer danza estableció como principio fundamental la búsqueda de la autenticidad expresiva a través del movimiento humano. Por eso se dice que "esta danza no sólo es un arte sino una forma de vivir" (GARAUDY, 2003, p. 15).

Aquí, una de las grandes influencias para los pioneros de la danza moderna (o libre) fue François Delsarte y sus proposiciones sobre el movimiento, la expresión corporal y el gesto. Algo que también fue de vital importancia para la creación del teatro pobre $^{12}$. De la influencia delsartiana se dice que,

es extraño que, sin haber creado un sistema, sin haber inventado un método, sólo por el hecho de haber descubierto unos principios, François Delsarte sea considerado hoy en día como el precursor de lo que se ha convertido en la base de la técnica de la danza moderna. La ciencia de Delsarte influyó la evolución de la expresión corporal que se propagó por los Estados Unidos a finales del siglo XIX. Los principios de este innovador se convertirían en las bases sobre las que reposan, después de casi un siglo, las distintas corrientes que animan la danza moderna. (BARIL, 1987, p. 364).

Ahora bien, con las bases de Delsarte y otras que fueron importantes para los pioneros de la danza moderna (ya se verá más adelante), se comenzó a gestar una nueva posibilidad de arte creativo. Una danza en la que el bailarín pudiera encontrarse a sí mismo y eliminar la tensión existente entre el mundo legitimado de lo clásico y la posibilidad de vivir la vida a través de la danza. Esta gestación

12 "He estudiado todos los métodos teatrales importantes de Europa y de otras partes del mundo. Los más importantes para mi propósito son los ejercicios rítmicos de Dullin, las investigaciones de Delsarte sobre las reacciones de extroversión e introversión, el trabajo de Stanislavski sobre las "acciones físicas», el entrenamiento biomecánico de Meyerhold, la síntesis de Vajtangov. También me fueron particularmente estimulantes las técnicas de entrenamiento del teatro oriental, específicamente la Ópera de Pekin, el Kathakali hindú, el Teatro No de Japón" (GROTOWSKI, 2004, p. 10). 
creativa, en su principio, tuvo tres exponentes importantes que ayudaron a sentar la bases que después consolidarían la danza moderna en los Estados Unidos. A continuación se abordan brevemente.

\section{El arte como pobreza: Isadora Duncan}

Lo que yo entiendo por bailar es liberar mi cuerpo a la salida del sol, sentir mis pies calzados con sandalias sobre la tierra, estar cerca de los olivos de Grecia y amarlos. (DUNCAN, 2003, p. 65).

La primera mujer que dedicó su vida a revivir el origen natural de la danza fue Isadora Duncan (San Francisco 1878 - Niza 1927). Ella desde muy pequeña "se rebeló contra la danza tradicional y se negó a aprender danza clásica, porque no aceptaba someterse a la disciplina del estudio de reglas estrictas" (BARIL, 1987, p. 25). Para Duncan, la danza debía regresar a su esencia y estar en consonancia con la naturaleza (con las olas, el viento, la tierra) no en contra de ella; tenía que ser una con el universo, para ello colocó su arte en el centro del cuerpo humano, en el plexo solar; con ello, la danza debía buscar la trascendencia del ser humano a través de la voluntad (idea que retoma de Shopenhauer). "La danza debía ser, por tanto, simplemente la gravedad natural de esta voluntad del individuo, que al final no es ni más ni menos que la traducción humana de la gravedad del universo" (SÁNCHEZ, 2003, p. 15).

Regresar a lo natural significaba desprenderse de todo aquello que le era superfluo. En un sentido grotowskiano, empobrecer la riqueza que estorbaba al arte de la danza para quedarse solamente con lo esencial. Observar la naturaleza para que se convierta en fuente de inspiración. Volver a lo natural, a lo esencial. Usar pies descalzos para mantener un contacto directo con la tierra. Reconectar con las raíces. Son algunas de las principales propuestas de empobrecimiento que utiliza Duncan. De ahí se deriva su filosofía, misma que ella define como el "alma» de las formas - esencia que busca en lo natural y en el arte de la cultura griega que define como la lengua de la humanidad ${ }^{13}$. Así, a diferencia de la danza clásica, Duncan va en busca de esa naturalidad. Lo natural que debía reflejarse también en el uso del vestuario, ya que para Isadora los atuendos del ballet eran modos de aprisionar al bailarín (por ejemplo, el uso de corsés, mallas y zapatillas). Razón por la que Isadora solía presentarse vestida con túnicas inspiradas en la cultura griega, sin calzado o con sandalias y con un telón de fondo o ciclorama de un solo color con el propósito de que todos sus movimientos pudieran ser apreciados sin ninguna distracción. Desnudar el escenario y mostrarse natural. Algo que también retoma el teatro pobre como parte de sus intereses fundamentales. Grotowski señala al respecto,

el interés fundamental es encontrar la relación apropiada entre el lector y el espectador en cada tipo de representación y cumplir la decisión mediante arreglos concretos. Abandonamos los efectos de luces [...]. Abandonamos el maquillaje [...]. La aceptación de la pobreza en el teatro, despojado de todo aquello que no le es esencial, nos reveló no sólo el meollo de ese arte sino la riqueza escondida en la naturaleza misma de la forma artística. ¿Por qué nos interesa el arte? Para cruzar nuestras fronteras, sobrepasar nuestras imitaciones, colmar nuestro vacío, colmarnos a nosotros mismos. No es una condición, es un proceso en el que lo oscuro dentro de nosotros se vuelve de pronto transparente. En esta lucha con la verdad íntima de cada uno, en ese esfuerzo por desenmascarar el disfraz vital, el teatro, con su perceptividad carnal, siempre me ha parecido un lugar de provocación. (GROTOWSKI, 2004, p. 16).

Así pues, de la misma manera que el teatro pobre, la danza de Isadora instaura las bases de su creación en lo esencial, en volver a lo natural, en llegar a la verdad íntima. Para 1904, Isadora Duncan establecería su primera escuela "cuyo objetivo sería encontrar la danza verdadera. De ningún modo una copia

13 "Sería incorrecto denominar mi arte griego. [...] Cuando se habla de arte griego en un sentido nacional estricto, ése no es mi arte. Yo pretendo hablar la lengua de la humanidad, no el dialecto de un pueblo" (DUNCAN, 2003, p. 127). 
de mi danza, sino el estudio de la danza en cuanto a arte" (DUNCAN, 2003, p. 82). Once años después en 1915 Ruth St. Denis y Ted Shawn abrirían las puertas del Denishawn una "escuela destinada a la enseñanza general de la danza, al estudio de los principales estilos y de las diferentes técnicas, especialmente de una técnica de danza [...] que se practica descalzo" (BARIL, 1987, p. 66). Es ahí donde se formarían los principales bailarines de la segunda generación, Martha Graham, Doris Humprey y Charles Weidman.

\section{El arte como encuentro espiritual: Ruth St. Denis y Ted Shawn}

Muchos protagonistas de la danza modernista como Ruth St. Denis, Ted Shawn, Isadora Duncan, Mary Wigman, Charlotte Bara y Martha Graham, entendieron el movimiento de la danza en términos extremadamente religiosos (ver Zander 2001). Mientras que por un lado escapaban de la límites religiosos institucionalizados, por el otro, desarrollaban una nueva religión a través de la danza. (SCHWAN, 2017, p. 28). ${ }^{14}$

\section{III.1 Ruth St. Denis}

Ruth St. Denis (Nueva Jersey 1878 - California 1968) es considerada junto con Isadora Duncan y Ted Shawn, iniciadora del movimiento moderno. Conocida también como la primera dama de la danza americana, "se ha dicho de ella que concibió un sistema totalmente nuevo y muy completo de movimientos: una escuela revolucionaria de la danza que en los Estados Unidos desencadenó una gran corriente estética" (BARIL, 1987, p. 48). Al igual que Duncan y gran parte de las mujeres de la época, Ruth St. Denis también estuvo influenciada por los principios de liberación femenina que recibe del contexto socio-histórico. Mismos que fueron reafirmados por influencia de su madre quien dejó de utilizar corsé y llevó a St. Denis a estudiar los principios de Delsarte, mismos que marcarían su vida como bailarina. En su etapa formativa Ruth St. Denis,

14 Trad. Propia. prueba en vano los cursos de danza clásica de Marie Bonfanti, con quien estudió Isadora Duncan. Ruth abandona rápidamente, incapaz de seguir las clases más allá del estudio de la tercera posición, sobre las cinco tradicionales de la danza clásica. Lo cual, no obstante, no le impide bailar de puntas, sacando buen partido de los consejos de Marwing, consistentes, principalmente en copiar las posiciones y las posturas de las estrellas de la danza, muy populares en aquella época. (BARIL, 1987, p. 49).

Con esto, la propuesta dancística de St. Denis se vio influenciada por lo clásico, ya que mantiene algunos de los pasos distintivos de este arte como las piruetas, los battements, los saltos, entre otros. Lo único que desecha por completo es el uso de las zapatillas de punta. Así, hasta aquí se pueden destacar dos elementos de eliminación en el sistema creado por St. Denis. Por un lado la búsqueda de la emancipación femenina a causa de la sumisión y represión social hacia la mujer; y por otro, la liberación física del pie del bailarín que hasta ese momento había vivido aprisionado con el uso de las zapatillas.

En adelante, su imaginario expresivo buscaría una liberación que la conectara con lo espiritual. De modo que, al igual que el teatro pobre su inspiración viene de relacionar la danza con el mundo de lo espiritual, de entenderlo como vía de encuentro con uno mismo y la espiritualidad. En el caso de St. Denis su inspiración venía de lo oriental, la mitología y la religión hindú comienzan a ser parte de su horizonte creativo. Su creación dancística se convirtió más que una forma de decir, en una forma de ser uno con Dios, en una forma de encuentro divino espiritual y de liberación.Para St. Denis "la danza es su verdadero medio de expresión artística, concentra toda su energía para alcanzar su única meta: hacer de la danza una fuerza espiritual para afrontar el mundo materialista de su época" (BARIL, 1987, p. 54). Es así como esta bailarina utilizó su arte como una vía de religación espiritual.

Aquí es importante decir que, un acontecimiento de la vida privada de Ruth definiría por completo el rumbo de la danza moderna en Estados Unidos: 
su matrimonio con Ted Shawn en 1914, suceso que permitió la aparición del Denishawn como espacio de formación en esta nueva forma de concebir el arte de la danza. Después, Ted y Ruth llevarían la danza moderna al lejano este en $1925^{15}$.

\section{III.2 Ted Shawn}

Ted Shawn (Missouri 1891 - Florida 1972) bailarín y coreógrafo que influyó de manera categórica en la creación de la danza moderna. Su inmersión en esta manifestación artística tuvo un inicio muy especial: La parálisis causada por un tratamiento contra la difteria hizo que se interesara -por recomendación de un médico- en la danza. "Fue entonces cuando se convirtió en alumno de Hazel Wallack, antigua alumna de Malvina Cavalazzi, la primera directora de la Escuela de ballet de la Metropolitan Opera de Nueva York" (BARIL, 1987, p. 57). De modo que la danza llega a Shawn por una enfermedad. Y con ello comienza a darse cuenta de lo que es el mundo de la danza y lo que significaba en esa época la presencia masculina en esa expresión artística. Así, mientras que la mujer de la época moderna luchaba por su reconocimiento en la vida social, Shawn luchaba por legitimar la figura masculina en el arte de la danza, ya que en ese momento estaba fuertemente representado por las mujeres. No es fortuito que la mayoría de sus coreografías -antes de la creación del Denishawn- fueran específicamente masculinas. Creaciones que ayudaron al empoderamiento de los hombres en el mundo de la danza.

Ahora bien, la propuesta dancística de Shawn -al igual que la de Duncan y St.Denis- estaba inspirada por los ejercicios físicos de Delsarte. Aunque para él era también importante mantener una relación íntima e intrínseca con la música y complementar la práctica con el conocimiento de otras

15 "Cuando Ruth St. Denis, Ted Shawn, y sus bailarines del Denishawn viajraon al lejano este en 1925, era la primera vez que un grupo americano de danza profesional "serio» se presentaba en Japón, China, India, Java, Burma, Malaysia, Manchuria y Ceylon. Esta gira contribuiría como patrón único al mosaico de la historia de la danza en nuestro país" (SHERMAN, 1976, p. 3). formas de danza, como folklore, danzas religiosas, étnicas, primitivas, entre otras, con el propósito de que el bailarín contara con un amplio repertorio de movimientos para su creación. Como parte del movimiento moderno de la danza, intrínsecamente inspirado en la espiritualidad y/o la religiosidad, Shawn creó un amplio repertorio inspirado en textos religiosos como su coreografía Joseph's Legend (1915). "También escribió y publicó extensivamente sobre la relación entre danza y religión [...] Shawn proclamó la danza como una forma legítima de expresión religiosa" (SCHWAN, 2017, p. 28-29) ${ }^{16}$. Además, como pedagogo Ted Shawn considera que,

la danza debe ser el núcleo de la educación escolar y universitaria, que es la raíz viva, carnal, de toda cultura. Es absurdo, dice, conducir a un niño al salón de clases y decirle: «ahora voy a formar tu inteligencia»; después llevarlo al gimnasio y decirle: «ahora voy a formar tu cuerpo»; después a la iglesia y decirle «ahora voy a formar tu alma». El hombre es uno. Dividirlo es mutilarlo. La verdadera educación debe formar a la vez un cuerpo dispuesto a moverse con facilidad y placer, para expresar y servir una vida voluntaria, una inteligencia lúcida, hábil en todos los mecanismos del pensamiento $y$, sobre todo, capaz en cada momento de plantear las preguntas fundamentales, de definir y escoger los fines de los actos y la vida, un corazón pleno de vida y de fuego, en el que las pasiones despierten la voluntad, que ame la belleza y encuentre su alegría en la lucha por una causa que lo trascienda. Estos tres aspectos de la vida única del hombre, según Ted Shawn, se encuentran en estrecha relación, y se distinguen sólo por medio de la abstracción. La danza les da la integración primordial y la realización cotidiana. (GARAUDY, 2003, p. 61-62).

Con esta última cita sólo queda decir que para Shawn la danza significaba una vía para la integración, para la religiosidad, un camino para amar la belleza, despertar la voluntad y trascender. Un modo de religar al ser humano, de integrarlo. Algo que fue muy importante en su momento para la concepción

\section{Trad. Propia.}


de la danza moderna como forma de encuentro espiritual humano y que de alguna manera retoma también el teatro pobre a mediados del siglo XX.

\section{Cierre (reflexiones finales)}

Con lo desarrollado en este ensayo y siguiendo los fundamentos del teatro pobre es posible decir que la danza moderna -en sus inicios, ya que la danza moderna y el teatro pobre sólo pueden ser concebidos como similares si se piensa en la danza moderna de los pioneros- es producto, tanto del método de eliminación como del empobrecimiento de las riquezas de la danza clásica de aquel momento. Ahora bien, la danza moderna es también producto del contexto social y de la visión individual de los pioneros. Todo ello en su conjunto (eliminación, empobrecimiento, contexto social y visión individual) ayudó como tierra fértil para concebir la danza moderna como camino para descubrir la vida espiritual humana, tal y como lo propone el teatro pobre. Pues "para Grotowsky la actuación es un vehículo. ¿Cómo decirlo? El teatro no es un escape, un refugio, una forma de vida, es un camino para descubrir la vida" (BROOK, 2004, p. 6).

Dicho de otra manera, la danza moderna y el teatro pobre no son fruto del destino sino de la riqueza, de la riqueza que presuponía vivir en el mundo de lo legitimado. Por esa abundancia normativa y técnica se opta por la eliminación, por la pobreza. Eliminar lo superfluo para quedarse con lo esencial. Esa es la necesidad que se impone de manera natural a ciertos personajes que consideran imposible seguir creando al margen de tales sistemas simbólicos impuestos por las instituciones sociales, artísticas. De modo que, como se pudo advertir, los fundadores del movimiento moderno en la danza moderna estadounidense estaban en contra de que ésta estuviera controlada por estereotipos corporales caducos, por cánones técnicos inflexibles y sistemas simbólicos restrictivos que no les permitían sentir y vivir plenamente la danza. Esto los llevó, vía el método de eliminación, a destruir lo anterior -lo existente, lo legitimado- y buscar nuevas formas de crear, de vivir, de simbolizar y de expresar la danza.
Aquí es importante poner atención en lo siguiente. Para legitimar la danza moderna ésta también tuvo que crear reglas y normas que aseguraran su preservación, tal y como lo hizo la escuela clásica en su momento. Aquí, la escuela del Denishawn, en comparación con el trabajo de Isadora Duncan, logró tener más éxito, ya que los bailarines más importantes de la segunda generación de la danza moderna fueron formados ahí, en el Denishawn. Sobre esto se puede decir que lograron la liberación de la institución clásica al adquirir cierta independencia en lo que constituiría el nuevo imaginario dancístico, paradójicamente, con el tiempo también se establecerían normas y reglas (algunas más inamovibles que otras).

Para cerrar, sólo queda decir que lo que aquí se presenta es simplemente un modo-entre muchos otros-, de explicar el nacimiento de una manifestación artística. En otras palabras, este trabajo ha de entenderse como un modo distinto de comprender la historia del surgimiento de la danza moderna en Estados Unidos. Un modo en el que danza y el teatro son analizados desde el recurso de la analogía de la pobreza.

\section{Referencias}

ANDERSON, Jack. The World of Modern Dance. Art Withouth Boundaries. lowa: University of lowa, 1997.

BARIL, Jacques. La danza moderna. Barcelona: Paidós, 1987.

BROOK, Peter. Prefacio. In: GROTOWSKI, Jerzy. Hacia un teatro pobre. Ciudad de México: Siglo XXI. pp. 5-8. 2004.

BURNS, James M.G. The Peace Movement. The Wilson Quarterly (1976-), 11 (2), pp. 184-184. 1987.

DALLAL, Alberto. La danza contra la muerte. Ciudad de México: Instituto de investigaciones estéticas / Universidad Nacional Autónoma de México. 1979.

DICKINSON, Edward R. Art, Women, Liberation 
en Dancing in the Blood: Modern Dance and European Culture on the Eve of the First World War. Cambridge: Cambridge University Press. pp 65-116. 2017.

DUNCAN, Isadora. El arte de la danza y otros escritos. Madrid: Akal. 2003.

ENRÍQUEZ, Eugene. La institución y las organizaciones en la educación y la formación. Buenos Aires: Novedades Educativas. 2002.

GARAUDY, Roger. Danzar su vida. Ciudad de México, México: Conaculta / Cenidi Danza. 2003.

GROTOWSKI, Jerzy. Hacia un teatro pobre. Ciudad de México: Siglo XXI. 2004.

KEETLEY, Dawn; PETTIGREW, John. Public women, public words: A documentary history of American feminism. Maryland: Rowman \& Littlefield. 2005.

MARIJAN, Milos. "Maga Magazinovic: The Main Concepts of Modern Dance." Accelerando Belgrade Journal of Music and Dance 3:8. Belgrado, Serbia. 2018.

MARTIN, John. The Modern Dance. Nueva York: Barnes. 1965.

REID, Ira D.A. Negro Movements and Messiahs, 1900-1949. Phylon (1940-1956). 10 (4), pp. 362-369. 1949.

SÁNCHEZ, J. A. "La danza liberada: el proyecto artístico de Isadora Duncan”. In: DUNCAN, Isadora. El arte de la danza y otros escritos, pp. 5-50. Madrid, España: Akal. 2003.

SCHWAN, Alexander. Ethos Formula: Liturgy and Rhetorics in the Work of Ted Shawn. Performance Philosophy, 3(1), 23-39. 2017.
SHERMAN, Jane. Soaring: the Diary and Letters of a Denishawn Dancer in the Far East, 1925-1926. Middletown, Conn: Wesleyan University Press. 1976

SORELL, Walter. Dance in its time. New York: Anchor Books, 1981.

SOTO, Carlos Pérez. Proposiciones en torno a la Historia de la Danza. Santiago de Chile, Chile: LOM. 2008.

Recebido: 15/06/2020

Aceito: $22 / 03 / 2021$

Aprovado para publicação: 13/04/2021

Este é um artigo de acesso aberto distribuído sob os termos de uma Licença Crea- tive Commons Atribuição 4.0 Internacional. Disponível em: <http://creativecommons.org/licenses/by/4.0>.

This is an open-access article distributed under the terms of the Creative Commons Attribution License 4.0 International. Available at: <http://creativecommons.org/licenses/by/4.0>.

Ce texte en libre accès est placé sous licence Creative Commons Attribution 4.0 International. Disponible sur: <http://creativecommons.org/licenses/by/4.0>. 